\title{
The Solar Spectral Irradiances from X-Ray to Radio Wavelengths
}

\author{
By ORAN R. WHITE \\ High Altitude Observatory, National Center for Atmospheric Research, \\ Boulder, CO 80307, USA
}

Sources of new measurements of the solar EUV, UV, and visible spectrum are presented together with discussion of formation of the solar spectrum as a problem in stellar atmospheres. Agreement between the data and a modern synthetic spectrum shows that observed radiative variability is a minor perturbation on a photosphere in radiative equilibrium and LTE. Newly observed solar variability in 1992 defines a magnetic episode on the Sun closely associated with changes in both spectral irradiances and the total irradiance. This episode offers the opportunity to track the relationship between radiation and magnetic flux evolution.

\section{Introduction}

Our understanding of the Sun's spectrum has improved substantially in the last two years because of progress in both theoretical synthesis of the spectrum and new observations. It is now possible to compute a synthetic solar spectrum that closely matches the existing measurements in the visible. New high precision UV irradiance observations beginning in 1991 will give a detailed time history of the UV solar spectrum between 115 $\mathrm{nm}$ and $410 \mathrm{~nm}$. A new experiment to measure the photospheric spectrum from $180 \mathrm{~nm}$ to $3200 \mathrm{~nm}$ referenced to a black body has just been flown by Thuillier and his colleagues. This is a major step toward knowing the absolute spectrum from the vacuum UV to the infrared. In 1992 the radiative output of the Sun apparently changed in response to a large scale decrease in the total magnetic flux in the photosphere, and this episode will be discussed as an example of the thermal and radiative consequences due to evolution of solar magnetism.

Here I address these topics to show not just progress but promise in our grasp of the solar spectrum and its variation. First I give a brief summary of new observations of spectral irradiance observations as well as discuss the formation of the solar spectrum as a problem in stellar atmospheres. As background for the empirical picture, the review by Lean (1991) and the book, Solar Radiation Flux, by E.A. Makarova, A.V. Kharitonov \& T.V. Kazachevskaya (1991) offer current compendia of measurements and their analyses. Individual measurements mentioned here are described more fully by the experimenters themselves in presentations at this meeting.

\subsection{New observations of spectral irradiance}

Figure 1 shows examples of irradiance measurements from the two extremes of the solar spectrum, X-ray and radio wavelengths. The first, $10.7 \mathrm{~cm}$ radio flux data, is our standard radiometric index of solar activity measured for the full solar disk (see Tapping \& Harvey 1994). The second, the X-ray irradiance between .5 and $3 \mathrm{~nm}$ is a new irradiance measurement constructed from 10 arcsec resolution solar images recorded continuously from space by the SXT instrument on the Yohkoh satellite (see Shibata 1994). The radio flux record shows solar variation over solar cycle 22 , while the new $\mathrm{X}$-ray record shows the change in solar radiative output from the chromosphere, transition region, and corona in 1991-93 as the Sun evolved from solar maximum in the declining phase of cycle 22 .

Our picture of the visible solar spectrum comes from several observers as discussed by 

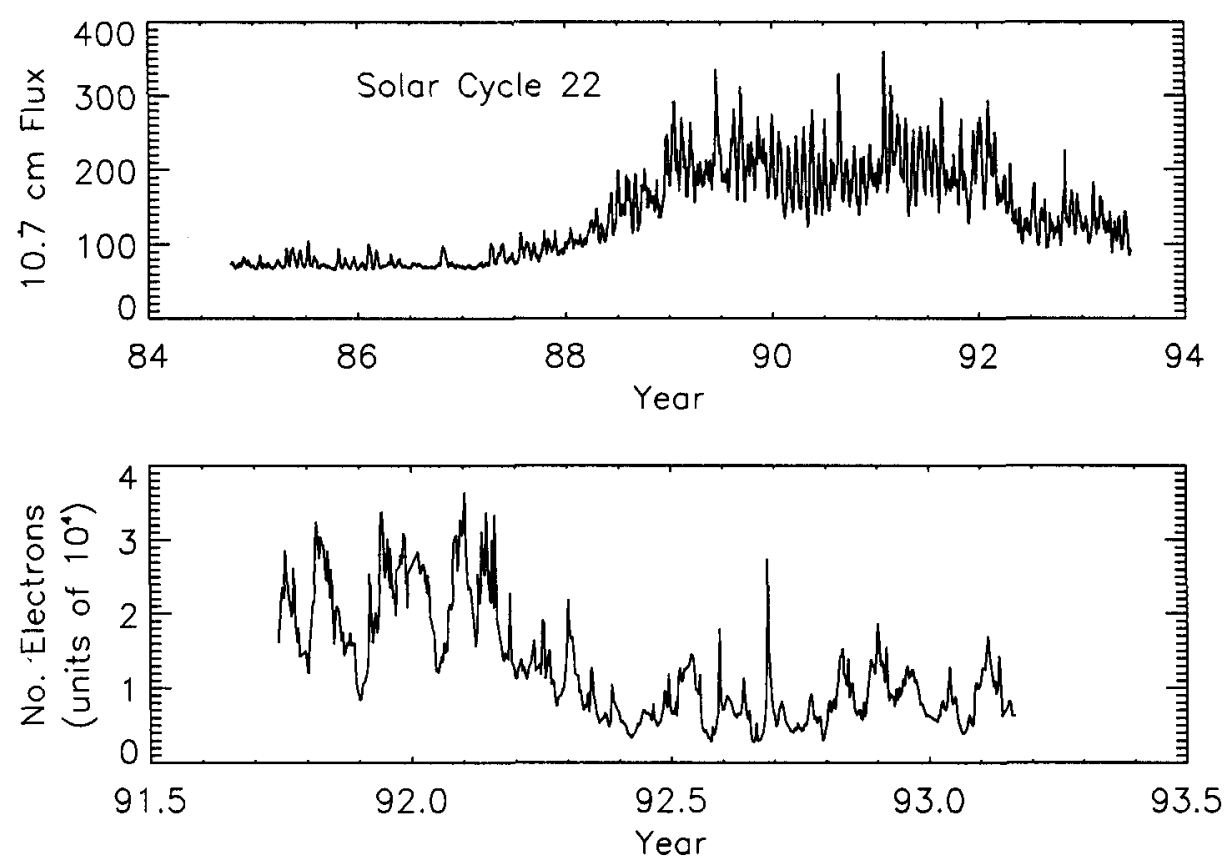

FIGURE 1. Upper panel: the $10.7 \mathrm{~cm}$ solar radio flux $\left(10^{-22} \mathrm{~W} / \mathrm{m}^{2}\right)$ in solar cycle 22 (Tapping \& Harvey 1994). Lower panel: daily mean X-ray irradiance from the Yohkoh/SXT X-ray imaging experiment, .5 to $2 \mathrm{~nm}$ (AlMg filter), data courtesy of L. Acton, G. Slater, and the Yohkoh project.

Makarova et al. (1994). Makarova and her colleagues show the precision of the mean of the best observations to be $\sim 3 \%$, which is still insufficient for detection of spectral variability corresponding to the $.1 \%$ change in total irradiance over the solar cycle. At wavelengths below $300 \mathrm{~nm}$, measurements come from a variety of satellites such as the Nimbus 7, Atmospheric Explorer E(AE-E), Solar Mesosphere Explorer (SME), Upper Atmosphere Research Satellite (UARS), the NOAA series (NOAA-9 and NOAA-11), and the Atlas series of shuttle flights. Occasional rocket-borne experiments that are recovered and recalibrated provide checks on the space instruments. The results from the many experiments before 1991 can be found in the review by Lean (1991).

The measurement picture changed in 1991 with the flight of the Solar Ultraviolet Spectral Irradiance Monitor (SUSIM) (Brueckner et al. 1994), the Solar Stellar Intercomparison Experiment (SOLSTICE) (Rottman et al. 1994), and the European Recoverable Carrier (EURECA) (Thuillier et al. 1994; Romero et al. 1994) instrument packages for comparison with the SBUV experiments on NOAA-9 and NOAA-11. These experiments are expected to give the highest accuracy and precision measurements of the solar UV spectrum between 115 and $420 \mathrm{~nm}$ ever made as well as new measurements of the solar spectrum from $180 \mathrm{~nm}$ to infrared. We now have for the first time five instruments recording the time history of the solar spectrum continuously from space. Data from these instruments are still in a preliminary state of analysis at this writing, but the early results suggest precisions of better than 1 percent at $300 \mathrm{~nm}$ in the SOLSTICE measurements. Table 1 summarizes these new experiments to measure spectral irradiance at radio, visible, and ultraviolet wavelengths. The Pioneer-Venus measurements discussed 


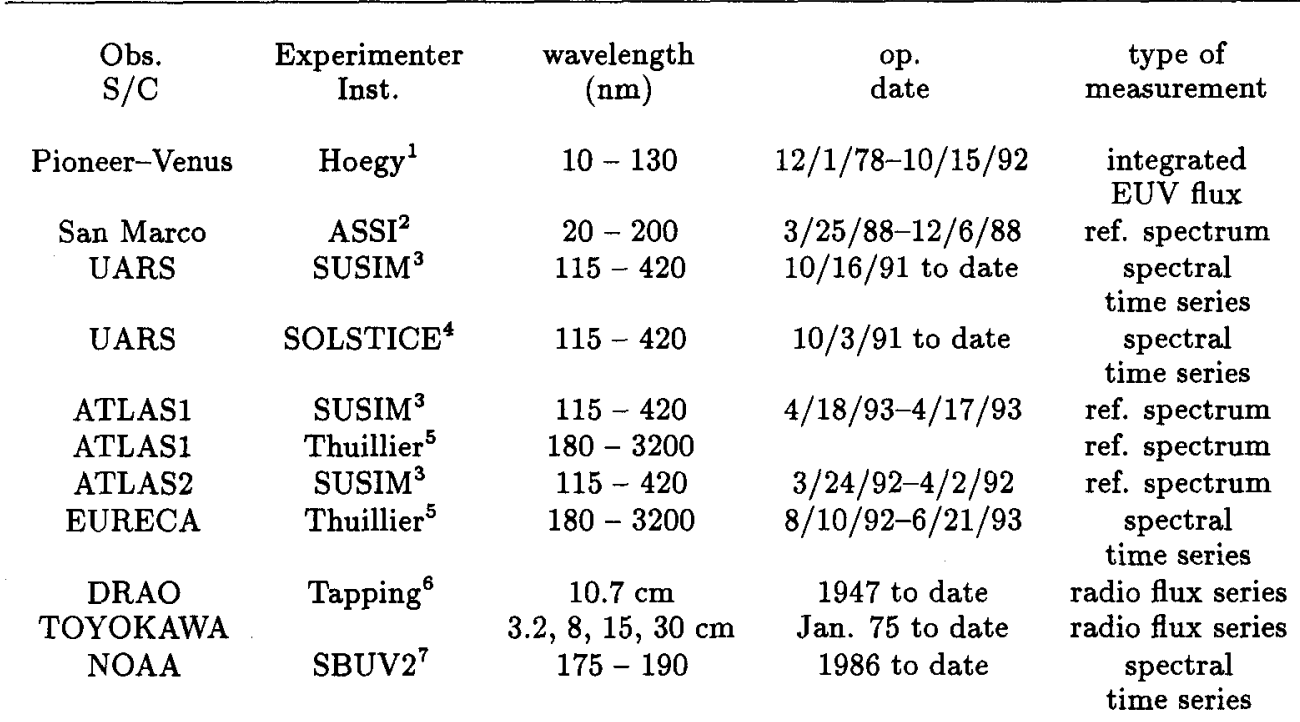

Refs: ${ }^{1}$ Pesnell \& Hoegy (1994); ${ }^{2}$ Schmidtke et al. (1992); ${ }^{3}$ Brueckner et al. (1994);

${ }^{4}$ Rottman et al. (1994); ${ }^{5}$ Thuillier et al. (1994); ${ }^{6}$ Tapping \& Harvey (1994);

${ }^{7}$ Cebula \& Deland (1992).

TABLE 1. Summary of new spectral irradiance observations.

by Pesnell \& Hoegy (1994) are unique since they are the only source of EUV flux time series since Hinteregger's AE-E experiments.

Photometrically calibrated imaging systems to record full disk solar images are the next step in understanding variation in the radiative output of the Sun, and Table 2 lists the systems producing data. Nishikawa (1990) and Chapman (1994) show that well calibrated images can yield relative irradiances that follow ACRIM I measurements. The San Fernando Observatory (SFO) system is designed specifically to determine the sources of variability due to sunspots and faculae. The images are made in the CaII $\mathrm{K}$ line and over a broad band at $672.3 \mathrm{~nm}$ (see Chapman 1994). Although designed for helioseismology, the National Solar Observatory (NSO) High L instrument yields very high quality images of the full disk in a $10 \AA$ band centered on the K line (see Harvey 1993, private communication). The Yohkoh images contain information about the irradiance for soft X-rays and, therefore, describe the variability of the radiative output of the solar corona and flares. The Big Bear Solar Observatory (BBSO) archive contains both $\mathrm{H}$ alpha and CalI $\mathrm{K}$ full disk images that serve as reference data (see Marquette 1992).

\section{What is the solar spectrum?}

We tend to think of the solar spectrum as a Planck function at a particular temperature, but the variation of opacity with wavelength plus the existence of temperature gradients in the outer solar atmosphere naturally lead to departures from a Planck function. A stellar spectrum can be thought of as a wavelength mapping of the source function at $\tau_{\lambda} \approx 2 / 3$. Figure 2 shows the solar spectrum together with a Planck function at $5770^{\circ} \mathrm{K}$. The large departures from the Planck function below $100 \mathrm{~nm}$ arise from the 


\begin{tabular}{|c|c|c|c|}
\hline $\begin{array}{l}\text { Obs. } \\
\text { S/C }\end{array}$ & $\begin{array}{l}\text { Experimenter } \\
\text { Inst. }\end{array}$ & $\begin{array}{l}\text { wavelength } \\
\quad(\mathrm{nm})\end{array}$ & $\begin{array}{l}\text { op. } \\
\text { date }\end{array}$ \\
\hline NAO & Nishikawa $^{1}$ & 545,770 & Dec. 87 - Apr. 88 \\
\hline YOHKOH & $\mathrm{SXT}^{2}$ & $.5-3$ & Aug. 91 to date \\
\hline $\mathrm{SFO}$ & CFDT $1,2^{3}$ & $393.1-393.5$ & $10 / 1 / 91$ to date \\
\hline SFO & CFDT $1,2^{3}$ & 672.3 & $10 / 1 / 91$ to date \\
\hline NSO & High $\mathrm{L}^{4}$ & $392.8-393.8$ & $3 / 2 / 93$ to date \\
\hline BBSO & CaII K ${ }^{5}$ & 393.5 & 1987 to date \\
\hline
\end{tabular}

Refs: ${ }^{1}$ Nishikawa (1990); ${ }^{2}$ Shibata $(1994) ;{ }^{3}$ Chapman $(1994) ;{ }^{4}$ Harvey (1993);

${ }^{5}$ Marquette (1992)

TABLE 2. Summary of photometric imager observations

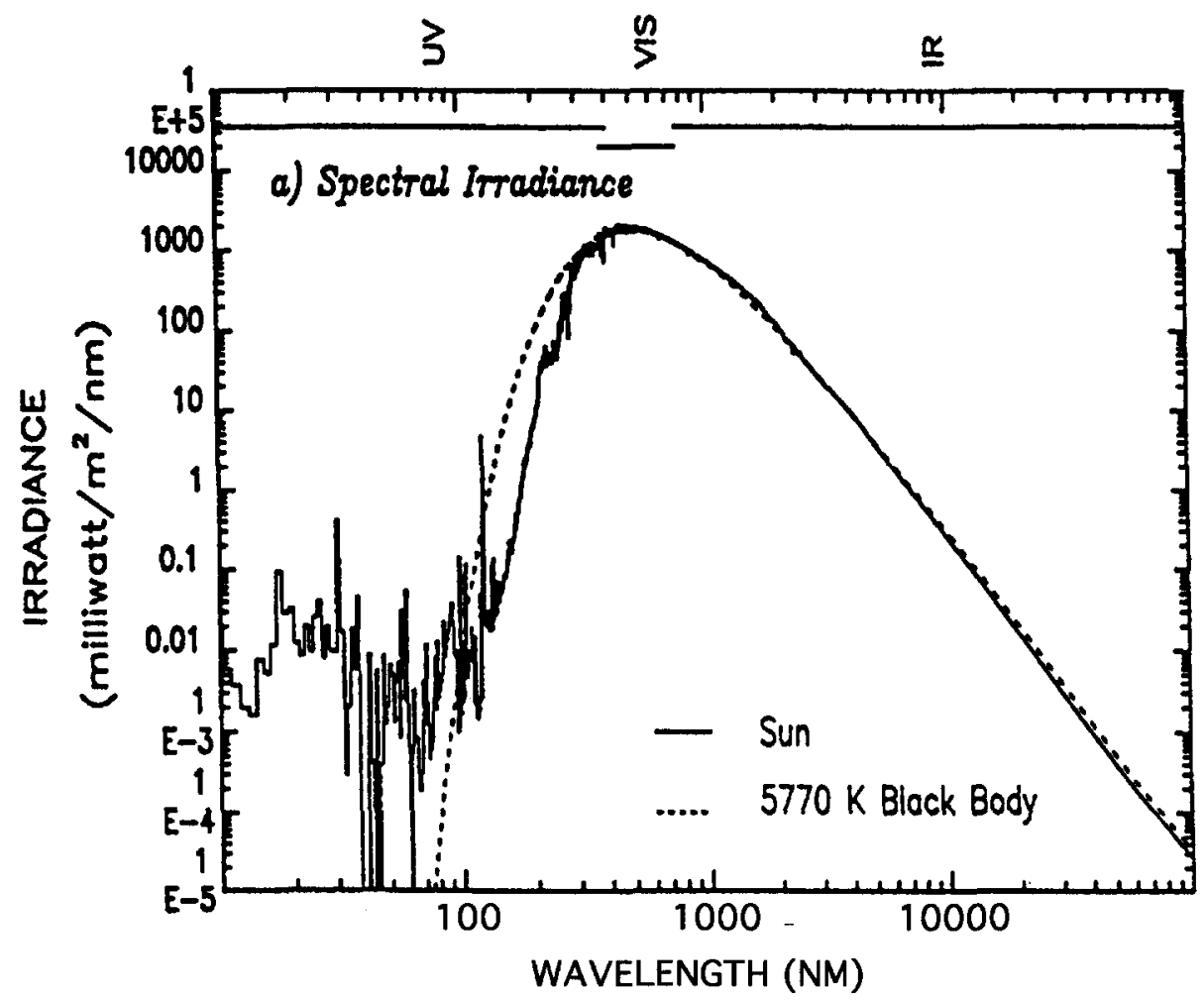

Figure 2. The solar radiative energy distribution compared to black body Planck function at $5770^{\circ} \mathrm{K}$, from Lean (1991).

existence of the chromosphere and corona while the departure between $150 \mathrm{~nm}$ and 450 $\mathrm{nm}$ arises from an increased photospheric opacity at these wavelengths.

The problem of how a star adjusts its emergent spectrum to carry the necessary flux is addressed in the theory of stellar atmospheres. In the case of a spherically symmetric photosphere where the energy flux is carried wholly by radiation; specification of the radiative flux, the chemical composition, the mass of the star and the opacity as function of temperature, density, and wavelength is sufficient to compute an emergent spectrum 


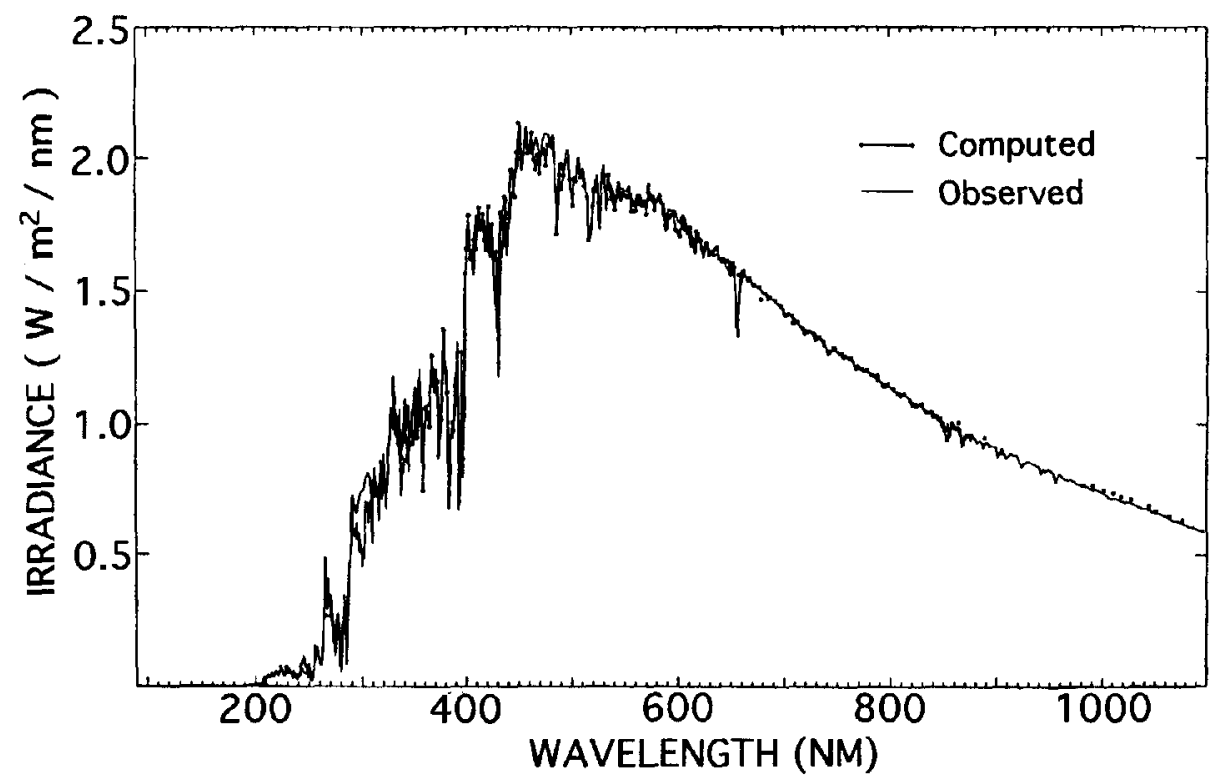

FIGURE 3. Comparison of synthetic solar spectrum computed by Kurucz (1991) with the measured spectrum from Neckel \& Labs (1984).

using a modern LTE stellar atmospheres code such as ATLAS developed by Kurucz (1991). Kurucz's contribution is the specification of some 58 million atomic and molecular transitions contributing to the opacity. The solar spectrum computed by Kurucz is shown in Figure 3 together with the empirical spectrum given by Neckel \& Labs (1984). Obviously, the shape of the computed spectrum follows the details of observed spectra well, which shows Kurucz' success in specifying free-bound absorption edges and overlapping spectral lines contributing to the opacity below $450 \mathrm{~nm}$. The effective temperature for this synthetic spectrum is $5771^{\circ} \mathrm{K}$.

The answer to my question 'What is the solar spectrum?' is 'In terms of the energy carrying capacity of the visible photosphere, the Sun's spectrum is formed in a quiet photosphere in LTE and radiative equilibrium.' Observationally, the Sun's total radiative output varies by only $1 / 1000$ th of the area under the spectral energy distribution curve shown in Figure 3.

The cause of radiative variability on the Sun is, to the first order, a small perturbation on the energy-carrying capacity of the outer layers where the bulk of the radiative energy originates. As stated by $\mathrm{H}$. Spruit in his presentation at this colloquium: the Sun's radiative variability appears to arise from changes in the surface emissivity due to the presence of magnetic fields, not from a uniform global fluctuation in the luminosity of the star.

\section{How to improve physical understanding of variability of the spectrum?}

A promising approach is the work of Avrett (1992) and Kurucz (1993) in the calculation of synthetic solar spectra for different stellar models corresponding to different types and mixes of the structures associated with solar activity. Such calculations will give us estimates of the change in 'color' of the solar spectrum, particularly below $450 \mathrm{~nm}$ where 


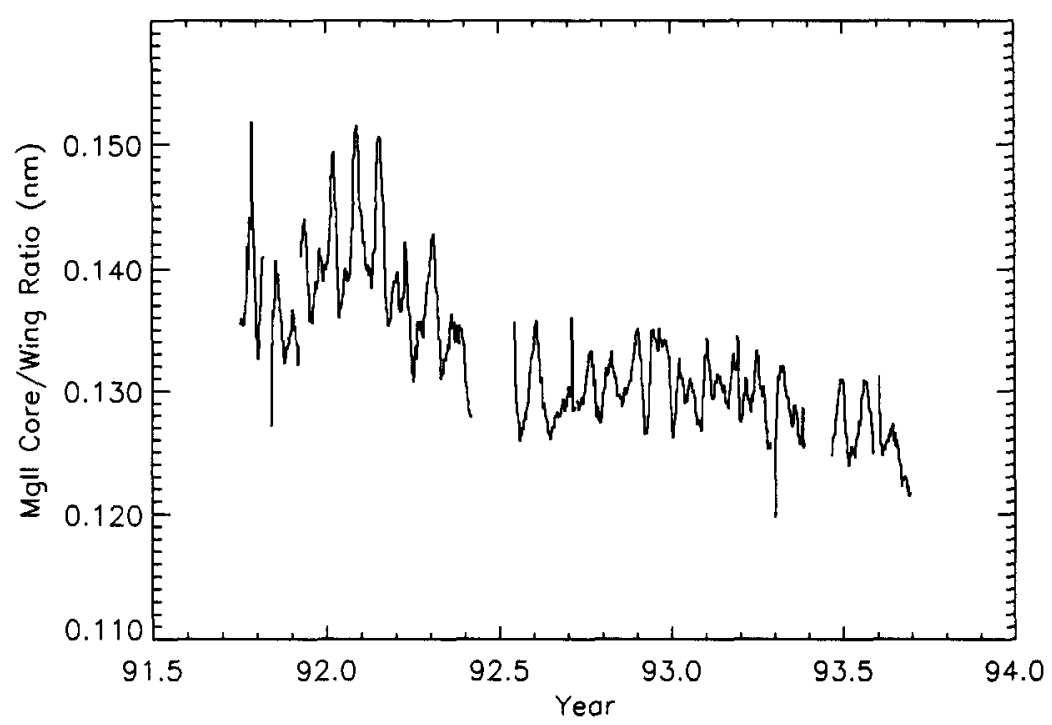

FIgURE 4. Plot of the MgII core/wing ratio for 1991 to 1993 from UARS/SOLSTICE spectral irradiance measurements. The core/wing ratio is a relative intensity measurement of the integrated chromospheric emission in the cores of the $280 \mathrm{~nm} \mathrm{MgII}$ doublet. From Rottman et al. (1994).

the effect of bound-bound line and free-bound continuum opacities change the shape of the spectral energy distribution.

Current measurements of spectral irradiances between $115 \mathrm{~nm}$ and $410 \mathrm{~nm}$ address the variability of this spectral region. The UARS measurements run from 3 October 1991 to date and now cover a range of solar activity from solar maximum levels to the descending phase of cycle 22, i.e., a period when the solar surface becomes less complicated. The interplay between these new observations and the spectrum synthesis will give insight into the cause of variation in the Sun's UV spectral energy distribution.

However, these approaches do not address the basic problem of the change in surface emissivity with the emergence and disappearance of magnetic flux in the photosphere. The question of the thermodynamics of evolving flux tubes and their effect on the radiative output is the core of the spectral variability problem as we see it today. The UARS and ground-based measurements in 1992 show a decline in chromospheric emission associated with a decrease in apparent magnetic field strength. This observed change offers the opportunity to see directly the relationship between radiative variability and solar magnetism on an almost global scale.

As an illustration of the radiative change observed in 1992, Figure 4 shows a plot of the $\mathrm{MgII}$ core/wing ratio as a function of time from the UARS (SOLSTICE) database. Beginning in early 1992 the MgII index and other chromospheric line emissions decline to a lower level by June 1992 and remain in this state since that time. Data from the National Solar Observatory (NSO) and Mt. Wilson Observatory (MWO) show that this radiative change occurred when the total apparent magnetic flux decreased by about a factor of two. Furthermore, the magnetic flux change occurs principally in the southern solar hemisphere where the number of active regions decreased markedly during the spring of 1992.

I illustrate this change in magnetism in Figure 5 from K. Harvey (1994) where she plots the total apparent magnetic flux in active regions for each Carrington rotation between 


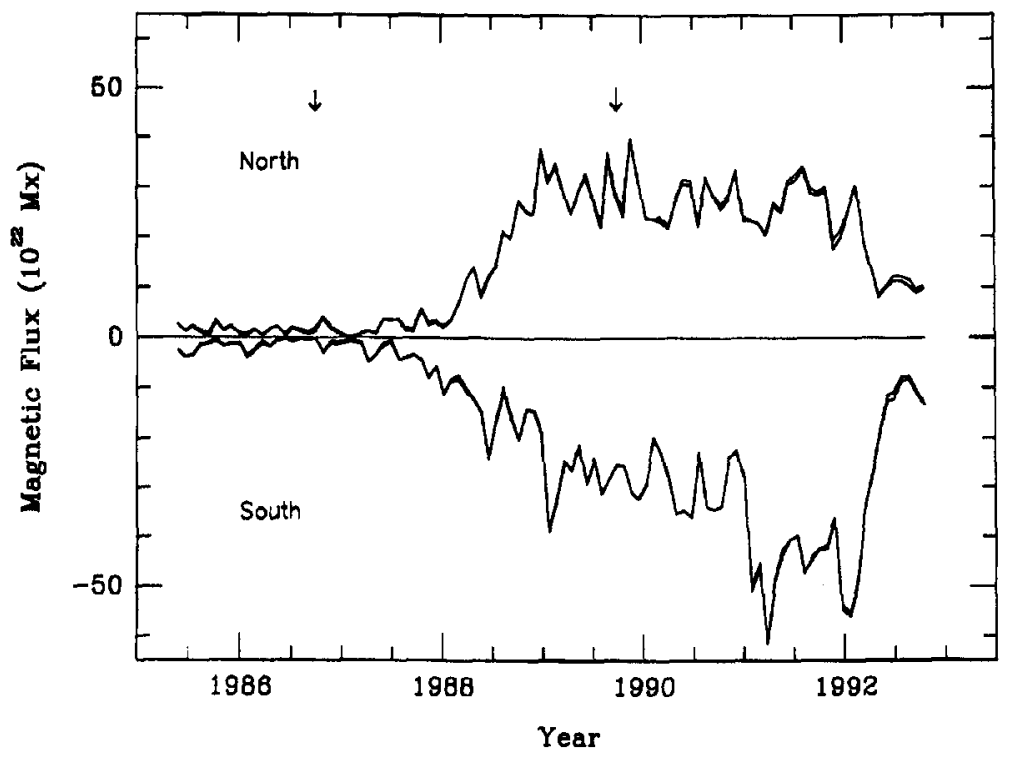

FigURE 5. The total mean magnetic flux in active regions during solar cycle 22. Note the rapid decline in the total solar magnetism in 1992. From K. Harvey (1994). Arrows mark solar cycle minimum and maximum.

1985.5 to 1992.7 . This plot shows that the magnetism in active regions decreased in both hemispheres to low levels almost globally around the Sun. This episode began in a period of high solar activity preceded by a rapid emergence of magnetic flux in the southern hemisphere during the last three months of 1991 . Thus, we see the radiative consequences of the emergence of flux in several active regions in the southern hemisphere followed by the temporary disappearance of the active region flux in both hemispheres in a period of nine months between October 1991 and June 1992.

What of the change in the integrated spectrum during this time? Four sets of total irradiance measurements are available during parts of this period: Earth Radiation Budget (Nimbus 7/ERB) from D. Hoyt and Lee Kyle (Hoyt et al. 1992), Active Cavity Radiometer (UARS/ACRIM II) from R.C. Willson (Willson 1994), and Solar Variability Experiment (EURECA/SOVA1 and SOVA2) from Crommelynk (Crommelynk et al. 1994) and from C. Fröhlich (Romero et al. 1994). Only the ERB data are available for detailed study at this time, and they are shown as the middle curve in Figure 6, together with the sunspot deficit computed from Photometric Sunspot Index (PSI) data supplied by J. Pap and C. Fröhlich. Correction of the ERB data for the effects of sunspots using the PSI time series gives the upper curve in Figure 6. This curve is then the estimate of the run of 'facular excess' on the Sun from the beginning of 1991 to January 1993 when the PSI database stops. This bright facular contribution began increasing by $\sim 1 \mathrm{Watt} / \mathrm{m}^{2}$ at the very end of 1991 , reached maximum near the first of February, and then steadily declined at the rate of $2 \mathrm{Watt} / \mathrm{m}^{2}$ per 6 months. We observe the decline for six months until the facular excess can no longer be computed with existing data.

I cautiously ask: Does this short history of the facular excess show the effect of thermal shadowing by large-scale flux tube aggregates lying below the visible surface late in 1991 followed by the rise and fall in facular excess as thermal energy carried by these magnetic aggregates appears and then steadily declines during the first six months of 1992 ? 


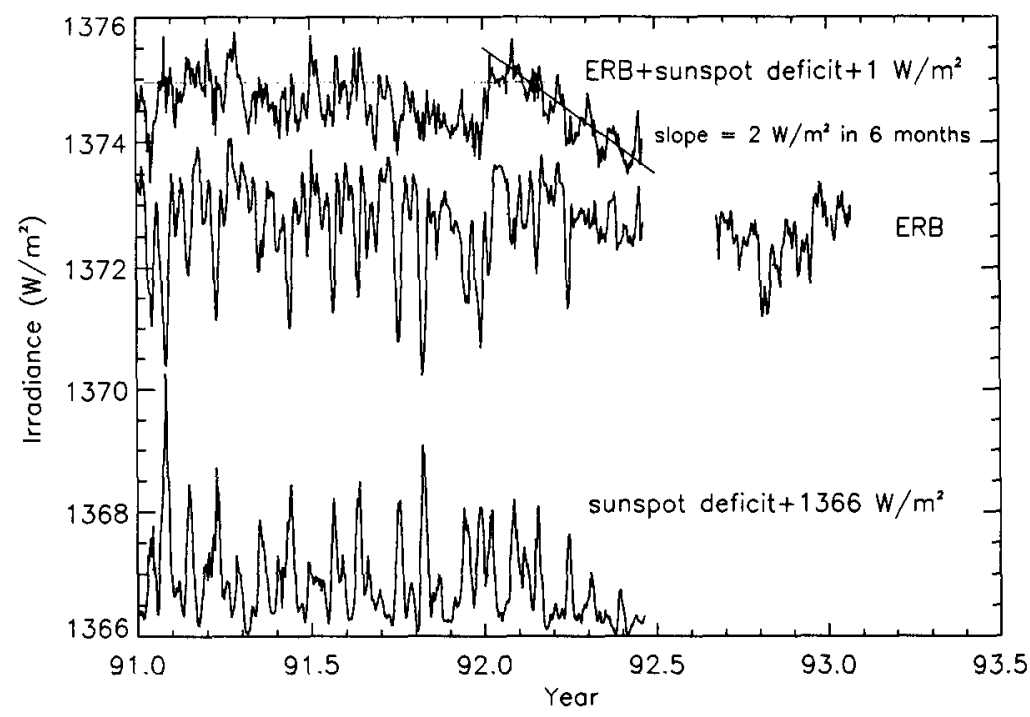

FiguRE 6. Comparison of the total irradiance variation from ERB in 1991 to 1993 with the sunspot deficit, and the total irradiance corrected with PSI. Upper curve: ERB+sunspot deficit$1 \mathrm{~W} / \mathrm{m}^{2}$, middle curve: ERB data uncorrected, lower curve: sunspot deficit $+1366 \mathrm{~W} / \mathrm{m}^{2}$. ERB data courtesy of Hoyt and Kyle. PSI data courtesy of Pap and Fröhlich.

Acknowledgments. I am privileged to have many generous colleagues who contributed both data and ideas to this paper. I particularly appreciate contributions from Dick Donnelly, Doug Hoyt, Peter Fox, Judit Pap, Claus Fröhlich, Dick Willson, Karen Harvey, Jack Harvey, Roger Ulrich, Loren Acton, Greg Slater, Tom Woods, Gary Rottman, Bernard Durney, B.C. Low and Gary Chapman. This research is supported in part by contract NASW-4721. The National Center for Atmospheric Research is sponsored by the National Science Foundation.

\section{REFERENCES}

Avrett, E. H. 1992 Temporal variations of near-UV, visible, and infrared spectral irradiance from a theoretical viewpoint. In Proceedings of the Workshop on the Solar Electromagnetic Radiation Study for Solar Cycle 22 (ed. R. Donnelly). pp. 20-42. NOAA ERL SEL, Boulder, CO, USA.

Brueckner, G. E., Floyd, L.E., Lean, J.L., Lund, P.A., Prinz, D.K. \& Van Hoosier, M.E. 1994 Irradiance observations from UARS/SUSIM and ATLAS/SUSIM In The Sun as a Variable Star: Solar and Stellar Irradiance Variations (ed. J.M. Pap, C. Fröhlich, H. S. Hudson \& S.K. Solanki). Cambridge University Press, in press.

Cebula, R. P. \& Deland, M. T. 1992 The SBUV-2 monitors and the NOAA-9 and NOAA11 satellites. In Proceedings of the Workshop on the Solar Electromagnetic Radiation Study for Solar Cycle 22 (ed. R.F. Donnelly). pp. 239-249. NOAA ERL SEL, Boulder, CO, USA.

Chapman, G. 1994 Full-disk ground-based photometric observations of the Sun. In The Sun as a Variable Star: Solar and Stellar Irradiance Variations (ed. J.M. Pap, C. Fröhlich, H. S. Hudson \& S. K. Solanki). Cambridge University Press, in press.

Crommelynck, D., Domingo, V., Fichot, A. \& Lee, R. 1994 Irradiance observations from the EURECA and ATLAS experiments. In The Sun as a Variable Star: Solar and Stellar Irradiance Variations (ed. J.M. Pap, C. Fröhlich, H. S. Hudson \& S. K. Solanki). Cambridge University Press, in press.

HaRvey, J. W. 1993 Private communication. 
HARVEY, K. \& TAPPING, K.F. 1994 Irradiance models based of solar magnetic field observations. Solar Phys., in press.

Pesnell, W.D. \& Hoegy, W.R. 1994 Solar cycle variations in the extreme ultraviolet. Solar Phys., in press.

Hoyt, D. V., Kyle, H.I., J.R. Hickey \& MAschhoff, R.H. 1993 The Nimbus 7 total irradiance: A new algorithm for its derivations. J. Geophys. Res. 97, A1, 5-63.

Kurucz, R. L 1991 New Lines, New Models, New Colors, Precision Photometery: Astrophysics of the Galaxy (ed. A.G.D. Philip, A.R. Upgren \& K.A. Janes). L. Davis Press, Schnectady, NY.

LEAN, J. 1991 Variations in the Sun's radiative output. Rev. of Geophys. 29, 4, 505-535.

Makarova, E. A., Kharitonov, A.V. \& Kazachevskoya, T.V. 1991 Solar Radiation Flux, Naulka Publishers, USSR.

Makarova, E. A., Kazachevskoya, T.V. \& Kharitonov, A.V. 1991 On the variability of some characteristics of solar flux. Solar Phys., in press.

Marquette, W. 1992 Calcium plage observations at Big Bear Solar Observatory. In Proceedings of the Workshop on the Solar Electromagnetic Radiation Study for Solar Cycle 22 (ed. R. Donnelly). pp. 154-159. NOAA ERL SEL, Boulder, CO., USA.

NeCKel, H. \& LABS, D. 1984 The solar radiation between $3300 \AA$ and 12,500 $\AA$. Solar Physics 909, 205-258.

NishiKawA, J. 1990 Estimation of total irradiance variations with the CCD solar surface photometer. Astrophys. J. 359, 235-345.

Romero, J., WeHrli, C. \& Fröhlich, C. 1994 Solar total irradiance variability from SOVA2 on board EURECA. Solar Phys., in press.

RotTman, G. J., Woods, T.N., White, O.R. \& LoNDON, J. 1994 Irradiance observations from the UARS/SOLSTICE experiment. In The Sun as a Variable Star: Solar and Stellar Irradiance Variations (ed. J. M. Pap, C. Fröhlich, H. S. Hudson \& S. M. Solanki). Cambridge University Press, in press.

Shibata, K. 1994 The variable structure of the X-ray corona as viewed by Yohkoh. In The Sun as a Variable Star: Solar and Stellar Irradiance Variations (ed. J.M. Pap, C. Fröhlich, H. S. Hudson \& S. K. Solanki). Cambridge University Press, in press.

Schmidtke, G., Woods, T.N., Worden, J., Rottman, G.J., Dolls, H., Wita, C. \& Solomon, S.C. 1992 Solar EUV irradiance from the San Marco ASSI: A reference spectrum. Geophys. Res. Letters 19, 21, 2175-2178.

TAPPING, K. F. \& HARVEY, K. 1994 Variations of coronal radiations at radio wavelengths. In The Sun as a Variable Star: Solar and Stellar Irradiance Variations (ed. J.M. Pap, C. Fröhlich, H. S. Hudson \& S.K. Solanki). Cambridge University Press, in press.

Thuillier, G., Hersé, M., Labs, D., Simon, P.C., Gillotay, D. \& Mandel, G. 1994 Irradiance observations in near-UV, visible, and near infrared spectral bands from measurements carried out during ATLAS-1 and EURECA-1 missions. In The Sun as a Variable Star: Solar and Stellar Irradiance Variations (ed. J. M. Pap, C. Fröhlich, H. S. Hudson \& S.K. Solanki). Cambridge University Press, in press.

WILlson, R. C. 1994 Irradiance observations from the Spacelab 1, SMM, UARS, and ATLAS experiments. In The Sun as a Variable Star: Solar and Stellar Irradiance Variations (ed. J. Pap, C. Fröhlich, H. S. Hudson \& S. Solanki). Cambridge University Press, in press. 